\title{
Inhibitory neurons can facilitate rhythmic activity in a neural network
}

\section{$\operatorname{AUTHOR}(\mathrm{S})$ :}

Shimokawa, T; Shinomoto, S

\section{CITATION:}

Shimokawa, T ... [et al]. Inhibitory neurons can facilitate rhythmic activity in a neural network. Physical Review E 2006, 73(6): 066221.

ISSUE DATE:

2006-06

URL:

http://hdl.handle.net/2433/49842

RIGHT:

Copyright 2006 American Physical Society 
PHYSICAL REVIEW E 73, 066221 (2006)

\title{
Inhibitory neurons can facilitate rhythmic activity in a neural network
}

\author{
Takeaki Shimokawa and Shigeru Shinomoto \\ Department of Physics, Graduate School of Science, Kyoto University, Sakyo-ku, Kyoto 606-8502, Japan
}

(Received 8 December 2005; revised manuscript received 8 May 2006; published 23 June 2006)

\begin{abstract}
Synchrony emerges in a population of oscillators interacting through in-phase couplings. We addressed a question of whether inhibitory neurons simply hinder the emergence of the synchronous activity among excitatory neurons, or facilitate it. An analysis of a simple phase model revealed that both cases may take place. Numerical simulations of the more realistic models revealed that inhibitory neurons rather facilitate rhythmic activity.
\end{abstract}

DOI: 10.1103/PhysRevE.73.066221

PACS number(s): 05.45.Xt, 87.18.Sn, 87.19.La

\section{INTRODUCTION}

In spite of the strong tendency of neurons toward mutual synchrony, collective synchronous activity is not ubiquitously seen in the healthy brain; only in such limited areas as the cat visual sensory area [1] and the olfactory bulb [2] is synchrony observed. Extensive synchronization takes place globally only in rather pathological conditions such as Parkinsonian tremor or epilepsy, in which the desynchronization is a matter requiring attention [3]. It has nevertheless been anticipated that synchronous activity plays an important role in signal transmission in the brain [4-6]. In order for the synchrony to be useful, the cooperation among neurons must be something that can be precisely controlled.

The neocortex consists of excitatory and inhibitory neurons. In understanding the dynamical cooperation and competition between the excitatory and inhibitory neurons, we address here a question of whether the presence of inhibitory neurons hinders the emergence of synchronous activity among the excitatory neurons, or facilitates it.

The emergence of synchronous activity in a population of heterogeneous oscillators has been demonstrated using the phase model [7-10]. The interaction function introduced in the original "Kuramoto model" is $-K \sin \phi$ that minimally satisfies the $2 \pi$ periodicity with respect to the difference between the phases of the internal state of interacting oscillators, $\phi$. The unavoidable delay in the signal transmission can be incorporated by introducing a phase shift, $\alpha$, into the interaction function as $-K \sin (\phi+\alpha)$. The dynamics of a population of oscillators interacting uniformly through couplings of this form were analytically solved by Sakaguchi and Kuramoto [11]. Numerical simulations of locally coupled oscillators revealed the emergence of spiral and target patterns that resemble those observed in real excitable media $[12,13]$. Since then, a number of theoretical studies have been carried out on the phase model [14-16]. Recent neurophysiological experiments have shed light on the phase response (resetting) characteristics of biological neurons [17,18]. Galan et al. discussed the relationship between the qualitative shape of the experimentally obtained phase response curve and the corresponding type of bifurcation leading to periodic behavior in neural oscillators [19].

In the present paper, the cooperation and competition between excitatory and inhibitory populations is investigated; first by analytically solving the models having simple sinu- soidal interaction functions; second via numerical simulations of models having more realistic phase interaction functions that incorporate the phase response characteristics of type 1 and type 2 neurons. An analysis of the simple model revealed that inhibitory neurons may either suppress or facilitate the synchronous activity. The synchrony is robustly controlled by the global parameters such as the coupling strength and the frequency difference between excitatory and inhibitory neurons. Numerical simulations of the more realistic models revealed that inhibitory neurons rather mostly facilitate the synchrony.

\section{ANALYSIS OF A SIMPLE PHASE MODEL}

First, we analytically solve the dynamics of a population of excitatory and inhibitory oscillators interacting through sinusoidal phase coupling functions, $\Gamma(\phi)=-K \sin (\phi+\alpha)$ with a common phase shift $\alpha$. For convenience, we call the oscillators interacting through a positive weight $K_{e}>0$ the "excitatory neurons," and those with a negative weight $-K_{i}$ $<0$ the "inhibitory neurons" (see Fig. 1).

The phase of an individual oscillator obeys the evolution equation

$$
\begin{aligned}
\frac{d \psi_{a}}{d t}= & \omega_{a}-\sum_{b \in \mathrm{ex}} \frac{K_{e}}{N} \sin \left(\psi_{a}-\psi_{b}+\alpha\right) \\
& +\sum_{b \in \mathrm{in}} \frac{K_{i}}{N} \sin \left(\psi_{a}-\psi_{b}+\alpha\right),
\end{aligned}
$$

where $\omega_{a}$ represents the original frequency of the oscillator.

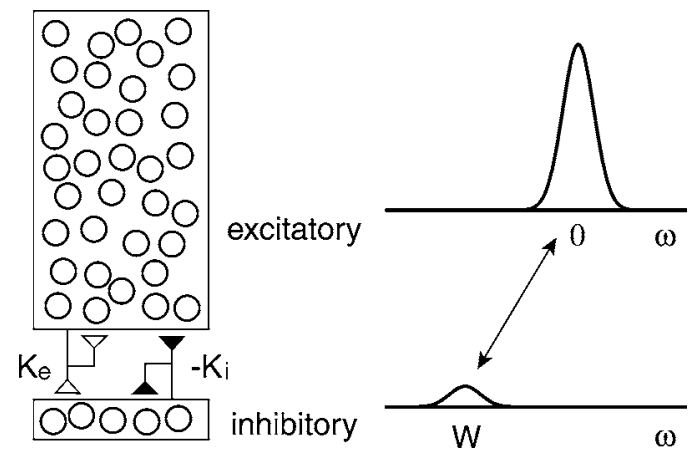

FIG. 1. A network consisting of excitatory and inhibitory neurons with their frequencies distributed around different mean values. 
The original frequencies of excitatory and inhibitory neurons are distributed according to $g_{e}(\omega)$ and $g_{i}(\omega)$, whose sum is normalized as $\int\left[g_{e}(\omega)+g_{i}(\omega)\right] d \omega=1$. The order parameters of excitatory and inhibitory neurons are defined as

$$
\begin{aligned}
\sigma_{e} \exp \left(i \Theta_{e}\right) & =\frac{1}{N_{b}} \sum_{b \in \mathrm{x}} \exp \left(i \psi_{b}\right), \\
\sigma_{i} \exp \left(i \Theta_{i}\right) & =\frac{1}{N_{b}} \sum_{b \in \mathrm{in}} \exp \left(i \psi_{b}\right) .
\end{aligned}
$$

Assuming that these order parameters evolve with identical frequencies and fixed phase lag $\Theta_{e}=\Omega t=\Theta_{i}+\theta$, the evolution equation (1) can be rewritten as an equation of a phase variable, $\Psi \equiv \psi-\Theta_{e}+\alpha$,

$$
\frac{d \Psi_{a}}{d t}=\omega_{a}-\Omega-A \sin \left(\Psi_{a}+B\right),
$$

where $A$ and $B$ are defined by $A e^{i B}=K_{e} \sigma_{e}-K_{i} \sigma_{i} e^{i \theta}$.

Oscillators having original frequencies $\left|\omega_{a}-\Omega\right| \leqslant A$ are entrained to the collective mode, with the phases being locked to the values that satisfy $\omega_{a}=\Omega+A \sin \left(\Psi_{a}+B\right)$. The distribution of the phases can be decomposed into those of the excitatory and inhibitory neurons as $n(\Psi)=n_{e}(\Psi)$ $+n_{i}(\Psi)$. Each component distribution is further decomposed into distributions consisting of the synchronized and desynchronized oscillators, $\quad n_{\{e, i\}}(\Psi)=n_{\{e, i\}}^{s}(\Psi)+n_{\{e, i\}}^{d s}(\Psi)$. The phase distribution of synchronized oscillators is

$$
n_{\{e, i\}}^{s}(\Psi)=g_{\{e, i\}}[\omega(\Psi)]\left|\frac{d \omega}{d \Psi}\right|,
$$

which is explicitly written as

$$
n_{\{e, i\}}^{s}\left(\Psi^{\prime}\right)= \begin{cases}g_{\{e, i\}}\left(\Omega+A \sin \Psi^{\prime}\right) A \cos \Psi^{\prime} & \left(\left|\Psi^{\prime}\right|<\frac{\pi}{2}\right), \\ 0 & \left(\left|\Psi^{\prime}\right|>\frac{\pi}{2}\right),\end{cases}
$$

where $g_{\{e, i\}}(\omega)$ is either $g_{e}(\omega)$ or $g_{i}(\omega)$, and $\Psi^{\prime}=\Psi+B$.

Oscillators with original frequencies $\left|\omega_{a}-\Omega\right|>A$ are not entrained to the collective mode. The distribution of the phases of those desynchronized oscillators is

$$
n_{\{e, i\}}^{d s}\left(\Psi^{\prime}\right)=\int_{|\omega-\Omega|>A} \frac{g_{\{e, i\}}(\omega)}{2 \pi} \frac{|\omega-\Omega| \sqrt{1-\left(\frac{A}{\omega-\Omega}\right)^{2}}}{\left|\omega-\Omega-A \sin \Psi^{\prime}\right|} d \omega .
$$

Using the relation of $A e^{-i B}=K_{e} \sigma_{e}-K_{i} \sigma_{i} e^{-i \theta}$, the selfconsistent equations (2) and (3) can be summarized as

$$
A=e^{-i \alpha} \int_{0}^{2 \pi}\left[K_{e} n_{e}\left(\Psi^{\prime}\right)-K_{i} n_{i}\left(\Psi^{\prime}\right)\right] \exp \left(i \Psi^{\prime}\right) d \Psi^{\prime} .
$$

The real number $A$ can be decomposed into the synchronized part, (a)

(b)
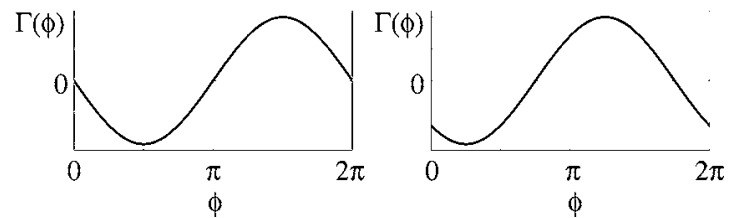

FIG. 2. Sinusoidal interaction functions: (a) $\Gamma(\phi)=-K \sin \phi$. (b) $\Gamma(\phi)=-K \sin (\phi+0.4 \pi)$.

$$
A^{s}=A e^{-i \alpha} \int_{-\pi / 2}^{\pi / 2} G\left(\Omega+A \sin \Psi^{\prime}\right) \cos \Psi^{\prime} \exp \left(i \Psi^{\prime}\right) d \Psi^{\prime}
$$

and the desynchronized part,

$$
A^{d s}=A i e^{-i \alpha} \int_{A}^{\infty} \frac{\{G(\Omega+x)-G(\Omega-x)\}}{x+\sqrt{x^{2}-A^{2}}} d x
$$

where $G(\omega) \equiv K_{e} g_{e}(\omega)-K_{i} g_{i}(\omega)$.

We solved these self-consistent equations to obtain the order parameters, $A=A^{s}+A^{d s}, B, \sigma_{e}, \sigma_{i}, \theta$, and $\Omega$, for the populations of oscillators interacting through simple sinusoidal couplings depicted in Figs. 2(a) and 2(b). For simplicity's sake, we assumed the interactions of excitatory and inhibitory neurons to be of the same strength and the opposite sign; $K\left(=K_{e}=K_{i}\right)$. Figures 3(a) and 3(b) depict the contour plots of the order parameter $\sigma_{e}$ that quantifies the degree of synchronous activity among the excitatory neurons. Here, the fractions of excitatory and inhibitory neurons were chosen as 0.8 and 0.2 in accordance with neuroanatomical data [20,21]. Their frequency distributions are both Gaussian with standard deviation unity and means 0 and $W$, respectively. Note that the evolution equations of phases have the translational invariance with respect to time, and therefore the frequencies here have the meaning only in a relative sense. (a)

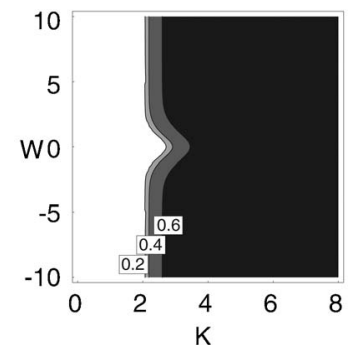

(b)

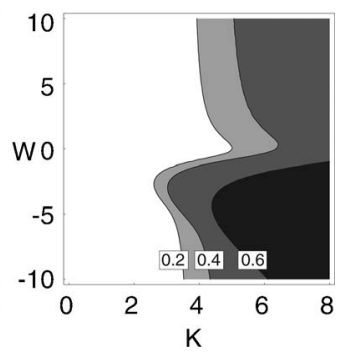

FIG. 3. Contour plots of the degree of synchrony $\sigma_{e}$, with a contour interval of 0.2 , in the space of parameters, coupling strength $K\left(=K_{e}=K_{i}\right)$ and the mean frequency of inhibitory neurons $W$ measured relative to that of excitatory neurons. (a) The case of vanishing phase shift, $\alpha=0$. Inhibitory neurons simply depress the collective activity, at $|W| \approx 0$. (b) The case of a positive phase shift, $\alpha=0.4 \pi$. Inhibitory neurons whose frequencies are distributed around negative frequencies (relative to that of the excitatory neurons) $W<0$ act on enhancing the synchronous activity. 
(a)
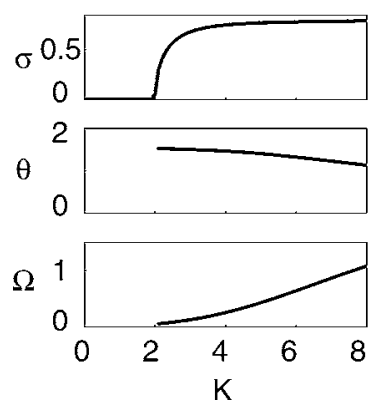

(b)

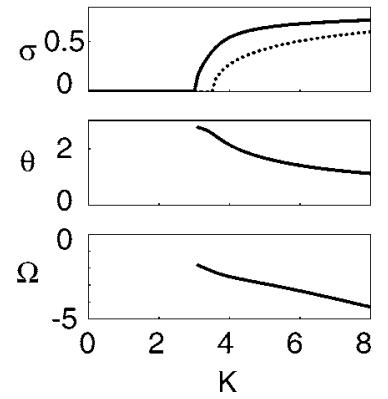

FIG. 4. Upper, (solid line) the degree of synchrony $\sigma_{e}$; (dotted line), the degree of synchrony of a network consisting of only the excitatory neurons of $80 \%$, with the inhibitory neurons of $20 \%$ being removed; middle, the phase lag of the inhibitory group $\theta$; lower, the frequency of the synchronized motion $\Omega$ measured relative to the original mean frequency of excitatory neurons. The mean frequency of inhibitory neurons is chosen as $W=-5$, relative to that of excitatory neurons. (a) The case of vanishing phase shift, $\alpha=0$. (b) The case of a positive phase shift, $\alpha=0.4 \pi$.

In the absence of the phase shift, $\alpha=0$, inhibitory neurons simply depress the collective activity, as is demonstrated by the dip of the contour near $|W| \approx 0$ in Fig. 3(a). However, in the presence of a positive phase shift, $\alpha>0$, the inhibitory neurons whose frequencies are distributed around negative frequencies $W<0$ (relative to that of excitatory neurons), enhance the synchronous activity, evidenced by the hump in Fig. 3(b), and vice versa. We also carried out numerical simulations of a population of oscillators with the same parameters. The order parameters estimated from the simulation fluctuate around the analytical solutions, but both are mutually consistent (data not shown).

Figures 4(a) and 4(b) demonstrate how the amplitude of the order parameter $\sigma_{e}$, the phase lag of the inhibitory group $\theta$, and the frequency of the resulting synchronized motion $\Omega$ depend on the coupling strength $K$, in the presence of the frequency lag of $W=-5$ in the inhibitory group. In the figures, the degree of synchrony $\sigma_{e}$ is compared with that of a network consisting of only the excitatory neurons of $80 \%$, with the inhibitory neurons of $20 \%$ being removed. It is observed from Fig. 4(b) that the synchrony is substantially enhanced by the addition of inhibitory neurons, in the case that the signal transmission delay is incorporated into the model.

It should be noted that even in the case of a positive phase shift, $\alpha>0$, there is a parameter region in which the synchrony is diminished by inhibitory neurons. The (rightward) dip of the contour near $|W| \approx 0$ relative to the contour near $|W| \approx 10$ in Fig. 3(b) indicates such situations: The case of a very large frequency difference such as $|W| \approx 10$ exhibits the situation similar to the case that inhibitory neurons are absent, because with a large $|W|$, inhibitory neurons "rotate" rapidly (relative to excitatory neurons) and their interactions are smoothed out. Therefore, the small value of $\sigma_{e}$ for $|W|$ $\approx 0$ in comparison with the value of $\sigma_{e}$ for $|W| \approx 10$ indicates the diminution of the synchrony. This nevertheless implies that rhythmic activity can be either facilitated or diminished according to global parameters such as the frequency difference between excitatory and inhibitory groups of neurons. (a)

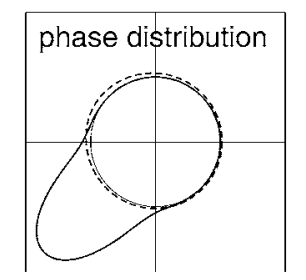

frequency distribution

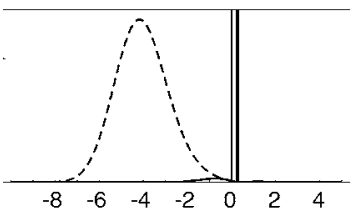

(1) (b)

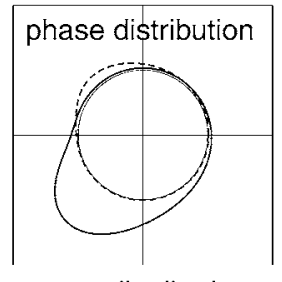

frequency distribution

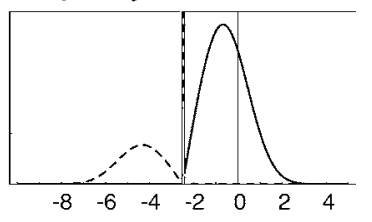

$\omega$
FIG. 5. Upper, phase distributions represented as ovals extended from a unit circle; lower, frequency distributions. The solid and dashed lines, respectively, represent the groups of excitatory and inhibitory neurons. (a) $\alpha=0, K=4$, and $W=-5$. (b) $\alpha=0.4 \pi, K=4$, and $W=-5$.

$\theta$ of about $\pi / 2$ in Fig. 4 indicates that a group of inhibitory neurons lags behind a group of excitatory neurons. The frequency of the resulting synchronized motion $\Omega$ is positive even in the presence of the frequency lag of $W=-5$ in the case of vanishing phase shift, $\alpha=0$, while it is negative in the case of a positive phase shift, $\alpha>0$. Figures 5(a) and 5(b) depict distributions of phases and frequencies of excitatory and inhibitory groups of neurons obtained with the presently developed analytical formula.

\section{SIMULATIONS OF THE MORE REALISTIC MODELS}

We thus observed the case in which "inhibitory neurons" facilitate synchronous activity among "excitatory neurons." However, the sinusoidal phase interaction function might be regarded as too simple to be considered realistic. We carried out numerical simulations of the phase models interacting through more realistic couplings in order to address this criticism. The phase interaction function $\Gamma(\phi)$ can be obtained from the phase response curve $Z(t)$ [9,17-19],

$$
\Gamma(\phi)=\frac{1}{T} \int_{0}^{T} Z\left(t+\frac{T \phi}{2 \pi}\right) s(t) d t,
$$

where $s(t)$ is the synaptic current typically given by an alpha function whose effective time scale is on the order of milliseconds. We approximate the type 1 and type 2 interaction functions as

$$
\begin{gathered}
\Gamma(\phi)=K[0.4-0.5 \sin (\phi+2.2)+0.1 \sin (2 \phi+2.8)], \\
\Gamma(\phi)=K[0.4-0.6 \sin (\phi+1.3)],
\end{gathered}
$$

which are, respectively, depicted in Figs. 6(a) and 6(b). Note that these phase interaction functions were given by moving the raw phase response curves to the left by an amount of about $0.2 \pi$ in a periodic interval of $2 \pi$, assuming that the 
(a)

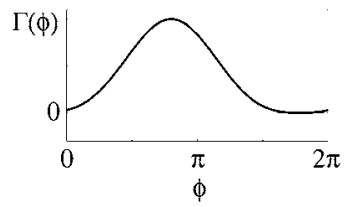

(b)

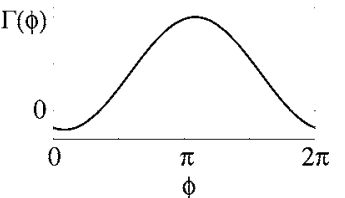

FIG. 6. Interaction functions: (a) type 1 interaction, Eq. (12); (b) type 2 interaction, Eq. (13). These "phase interaction functions" were given by moving the raw phase response curves to the left by $0.2 \pi$, by taking the signal transmission delay of a few milliseconds into account, with Eq. (11). See the text for details.

transmission delay caused by $s(t)$ in Eq. (11) to be of $\mathrm{ms}$ order, and the period of oscillation $T$ to be one order longer. The qualitative features of the following results were not altered even if the above phase interaction functions are moved back by $0.1 \pi$ or $0.2 \pi$ to the right; the latter of which corresponds to the absence of signal transmission delay. The inhibitory couplings are approximated here by reversing the sign of the excitatory couplings, as before.

We were interested in whether the presence of inhibitory neurons brings about the synchronous activity in the network. Figures 7(a) and 7(b) depict contour plots of the order parameters computed from the numerical simulations of those two models. In the numerical simulation, the numbers of excitatory and inhibitory neurons were, respectively, 800 and 200. It is observed from those plots that the synchronous activity is enhanced by the presence of inhibitory neurons. Note that the realistic phase response curves can be approximated by simply adding a constant to a sinusoidal function, as demonstrated in Fig. 6(b) of Eq. (13). Adding a constant to the interaction function simply induces the frequency shift simultaneously in all neurons in this uniform coupling model. Therefore, the constant term in the coupling function does not affect the emergence of synchrony, and therefore the self-consistent equation formula developed above can be directly applied to the model of Eq. (13). We have carried out both the analysis of self-consistent equations and the direct simulation of the evolution equations with respect to the type 2 model. Though the analysis of the self-consistent equations gave more accurate estimate of the order parameters, we demonstrated in Fig. 7(b) the results of numerical simulation.

Figures 8(a) and 8(b) demonstrate $\sigma_{e}, \theta$, and $\Omega$ in the presence of the frequency lag of $W=-5$ in the inhibitory (a)

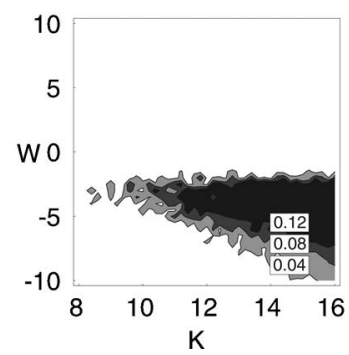

(b)

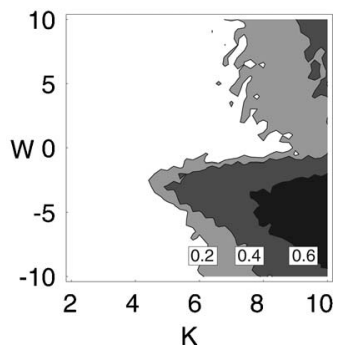

FIG. 7. Contour plots of the degree of synchrony $\sigma_{e}$. (a) Type 1 excitatory and inhibitory neural oscillators of Fig. 6(a). (b) Type 2 excitatory and inhibitory neural oscillators of Fig. 6(b). The contour intervals of (a) and (b) are, respectively, 0.04 and 0.2. (a)
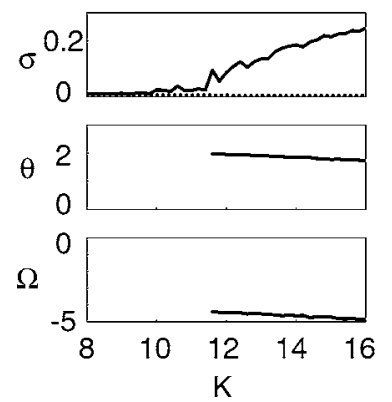

(b)
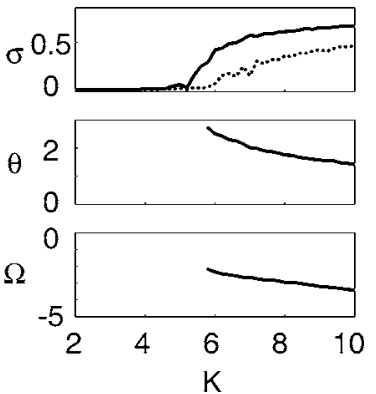

FIG. 8. Same as in Fig. 4; upper, (solid line) the degree of synchrony $\sigma_{e}$; (dotted line) the degree of synchrony of a network consisting of only the excitatory neurons of $80 \%$, with the inhibitory neurons of $20 \%$ being removed; middle, the phase lag $\theta$; lower, the frequency of the synchronized motion $\Omega$; the mean frequency of inhibitory neurons is chosen as $W=-5$, relative to that of excitatory neurons. (a) Type 1. (b) Type 2.

group, as in Figs. 4(a) and 4(b). In these figures, the degree of synchrony $\sigma_{e}$ is also compared with that of a network consisting of only the excitatory neurons of $80 \%$, with the inhibitory neurons of $20 \%$ being removed. No effective synchrony is observed in the network consisting of type 1 excitatory neurons alone, as represented by the dotted line in Fig. 8(a). This feature is consistent with the known fact that a network of type 1 excitatory neurons with no transmission delay does not exhibit a collective oscillation [22,23]. The solid line in Fig. 8(a) demonstrates that the synchrony emerges by adding the inhibitory neurons to the network consisting of type 1 excitatory neurons.

$\theta$ of about $\pi / 2$ in the Fig. 8 is common to that in Fig. 4, indicating that a group of inhibitory neurons lags behind a group of excitatory neurons. The frequencies of resulting synchronized motion $\Omega$ are both negative in two cases. Distributions of phases and frequencies of 800 excitatory neurons and 200 inhibitory neurons are depicted in Figs. 9(a) and 9(b). It is observed from Fig. 9(a) that the synchrony of type 1 network is mainly induced by inhibitory neurons. This (a)
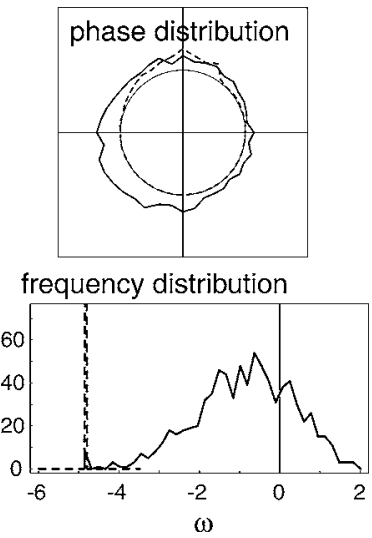

(b)

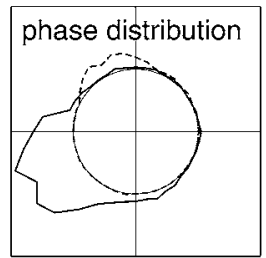

frequency distribution

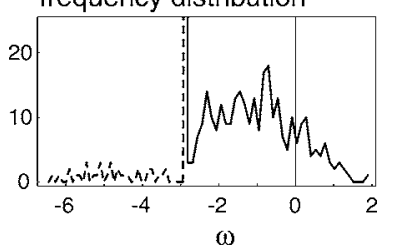

FIG. 9. Upper, phase distributions; lower, frequency distributions. The solid and dashed lines, respectively, represent those of excitatory and inhibitory neurons. (a) Type 1 network, with $K=16$ and $W=-5$. (b) Type 2 network, with $K=8$ and $W=-5$. 
situation is not altered by moving back the phase interaction function $\Gamma(\phi)$, Eq. (12), to the right by $0.1 \pi$, or $0.2 \pi$, the latter of which corresponds to the vanishing transmission delay.

\section{DISCUSSION}

In this paper, we have demonstrated that inhibitory neurons can either suppress or facilitate synchronous activity among excitatory neurons. Especially in the models having more realistic phase interaction functions, the presence of inhibitory neurons rather facilitates the appearance of rhythmic activity. Collective synchrony does not appear in a homogeneous population that consists of only type 1 excitatory neurons, but is materialized in a heterogeneous population that consists of excitatory and inhibitory neurons. The appearance of the rhythmic activity could be controlled by the global parameters such as the frequency difference of the groups of excitatory and inhibitory neurons. Note that the firing frequencies of biological inhibitory neurons are typically higher than those of excitatory neurons. This condition corresponds to $W>0$ in our model, indicating a tendency toward the desynchronization, which is achieved in the healthy brain. Our theory indicates that it is nevertheless possible that the synchrony is induced (temporally) in the network by decreasing the firing frequencies of inhibitory neurons.

In the present study, inhibitory couplings were approximated by reversing the sign of the excitatory couplings. It is desirable to examine wider range of functions that account for more realistic interactions, and furthermore, search for the general condition for the synchrony being facilitated by adding inhibitory neurons. It would be also interesting to examine the present theoretical prediction in a biological experiment by controlling the mean frequencies of excitatory and inhibitory neurons.

\section{ACKNOWLEDGMENTS}

The authors thank Yuichiro Sakamoto, Hiroya Nakao, and Kensuke Arai for helpful discussions. This study is supported in part by Grants-in-Aid and the 21st Century COE "Center for Diversity and Universality in Physics" from MEXT of Japan.
[1] C. M. Gray, P. König, A. K. Engel, and W. Singer, Nature (London) 338, 334 (1989).

[2] G. Laurent and H. Davidowitz, Science 265, 1872 (1994).

[3] C. Hauptmann, O. Popovych, and P. A. Tass, Biol. Cybern. 93, 463 (2005).

[4] M. Abeles, Corticonics: Neural Circuits of the Cerebral Cortex (Cambridge University Press, New York, 1991).

[5] M. Diesmann, M. O. Gewaltig, and A. Aertsen, Nature (London) 402, 529 (1999).

[6] A. D. Reyes, Nat. Neurosci. 6, 593 (2003).

[7] A. T. Winfree, J. Theor. Biol. 16, 15 (1967).

[8] Y. Kuramoto, in International Symposium on Mathematical Problems in Theoretical Physics, edited by H. Araki (SpringerVerlag, New York, 1975), Vol. 39 of Lecture Notes in Physics, pp. 420-422.

[9] Y. Kuramoto, Chemical Oscillations, Waves, and Turbulence (Springer-Verlag, New York, 1984).

[10] G. B. Ermentrout and N. Kopell, J. Math. Biol. 29, 195 (1991).

[11] H. Sakaguchi and Y. Kuramoto, Prog. Theor. Phys. 76, 576 (1986)
[12] H. Sakaguchi, S. Shinomoto, and Y. Kuramoto, Prog. Theor. Phys. 79, 1069 (1988).

[13] H. Sakaguchi, S. Shinomoto, and Y. Kuramoto, Prog. Theor. Phys. 77, 1005 (1987).

[14] T. Aonishi, K. Kurata, and M. Okada, Phys. Rev. Lett. 82, 2800 (1999).

[15] H. Daido, Phys. Rev. Lett. 87, 048101 (2001).

[16] J. A. Acebrón et al., Rev. Mod. Phys. 77, 137 (2005).

[17] B. S. Gutkin, G. B. Ermentrout, and A. D. Reyes, J. Neurophysiol. 94, 1623 (2005).

[18] T. I. Netoff et al., J. Neurophysiol. 93, 1197 (2005).

[19] R. F. Galán, G. B. Ermentrout, and N. N. Urban, Phys. Rev. Lett. 94, 158101 (2005).

[20] P. L. A. Gabbot and P. Somogyi, Exp. Brain Res. 61, 323 (1986).

[21] S. H. Hendry, H. D. Schwark, E. G. Jones, and J. Yan, J. Neurosci. 7, 1503 (1987).

[22] D. Hansel, G. Mato, and C. Meunier, Neural Comput. 7, 307 (1995).

[23] D. Hansel and G. Mato, Neural Comput. 15, 1 (2003). 